\title{
BROC Numa, La géographie de la Renaissance
}

(1420-1620)

Paris éditions du CTHS, format 82, 2019, 436 p.

Jean-Pierre Husson

\section{CpenEdition}

Journals

Édition électronique

URL : https://journals.openedition.org/geohist/1459

DOI : 10.4000/geohist. 1459

ISSN : 2264-2617

Éditeur

Association française de la Revue de géographie historique

Référence électronique

Jean-Pierre Husson, «BROC Numa, La géographie de la Renaissance (1420-1620) 》, Revue de géographie historique [En ligne], Comptes-rendus, mis en ligne le 20 mai 2020, consulté le 19 septembre 2021.

URL : http://journals.openedition.org/geohist/1459; DOI : https://doi.org/10.4000/geohist.1459

Ce document a été généré automatiquement le 19 septembre 2021.

\section{cc)}

Ce(tte) œuvre est mise à disposition selon les termes de la Licence Creative Commons Attribution -

Pas d'Utilisation Commerciale - Pas de Modification 4.0 International. 


\section{BROC Numa, La géographie de la Renaissance (1420-1620)}

Paris éditions du CTHS, format 82, 2019, 436 p.

Jean-Pierre Husson

\section{RÉFÉRENCE}

BROC Numa, La géographie de la Renaissance (1420-1620),

Paris éditions du CTHS, format 82, 2019, $436 \mathrm{p}$.

1 Le temps de la Renaissance incarne les balbutiements de la naissance de la géographie comme science actant et accompagnant l'ouverture sur les océans, la découverte du monde, sa configuration confirmée en sphère. Ce constat est un temps fort de l'humanisme traversé par des phases d'euphorie, de doutes, avec de mémorables joutes scientifiques à propos de la rotondité de la terre. Guerres de religions et solutions apportées par le concile de Trente évoquent un contexte de graves crises qui vont crescendo, avec par exemple la récupération de la cartographie au service de l'art de la guerre et de la poliorcétique. En rééditant dans un format pratique $(12 \mathrm{X} 18,5 \mathrm{~cm})$ l'ouvrage de Numa broc paru en 1986, les éditions du CTHS font preuve d'une heureuse initiative. Cette lecture sera utile à tous ceux qui aiment la géographie, souhaitent connaître sa genèse et encore les façons d'élaborer les cartes anciennes. Numa Broc est à la fois historien et géographe. Il accorde autant d'importance aux échelles croisées des temporalités et des espaces. Il fut un des rares géographes à prendre pour posture de travailler dans le labyrinthe du passé sans vouloir obligatoirement raccorder ce dernier à l'actuel. Pour lui, le temps la Renaissance fut un objet d'étude en soi. Il suit le père François de Dainville, entre dans la catégorie des géographes humanistes, savants, érudits. Il rapproche les territoires, les échelles, les récits de voyages et sait souligner la poésie des lieux. N. Broc s'est passionné pour la trilogie associant la cosmographie, la chorographie (ou topographie) et la cartographie. Son inventaire des cartes et portulans traduit une impression heureuse et colorée des productions faites pendant la 
Renaissance. Sa plongée dans les $\mathrm{XV}^{\mathrm{e}}$ et $\mathrm{XVI}{ }^{\mathrm{e}}$ siècles. Montre des temps de tâtonnements, d'hésitations malgré les découvertes et voyages. Ces derniers sont un volet primordial de la Renaissance. La période des grandes découvertes s'entoure d'un halo d'imaginaire pour accompagner les récits des voyageurs. La géographie reste cependant sous l'ombre tutélaire de Ptolémée. Textes, lettres et récits de voyages sont repris, mis en forme par les géographes de cabinets. Marins, mécènes et cartographes ont placé leurs différents talents au service d'une œuvre commune; la découverte progressive des terres émergées, des océans paisibles ou rugissants et encore des solitudes englacées. Une perception inquiète de la part respective de l'écoumène et des zones arides, hostiles est redéfinie.

2 Organisé dans la succession de quinze chapitres, ce livre retient une trame chronologique qui s'impose. Il s'agit de voyager dans deux siècles de profonds changements provoquant l'élargissement des territoires progressivement identifiés, reconnus, appropriés. L'ouvrage intègre les temps d'assimilation des découvertes, tient compte des silences et des rétentions d'informations liées au secret qui pèse sur les conquêtes et l'établissement des routes commerciales où sont espérés des profits considérables. Derrière la géographie de la Renaissance se profilent des stratégies à la fois portées par la course à la construction des premiers empires et l'égrenage des comptoirs portuaires où transitent des marchandises précieuses (épices, soie, porcelaine, or et argent, etc.).

3 Si l'écriture est limpide, le contenu du volume est savant, dense, très érudit. Sa lecture ne peut être envisagée comme une découverte initiale du sujet. Les chapitres sont distribués entre quatre grandes orientations thématiques. Celles-ci sont établies dans des approches croisées entre les découvertes, la construction des empires et la confection de cartes manuelles ou/et dupliquées grâce à l'essor récent de l'imprimerie. Ces beaux objets sont à la fois œuvre d'art et de sciences. Les cartes se répartissent en trois familles de produits : les cosmographies, les chorographies et les cartes. Ajoutons les portulans progressivement resautés de quotités angulaires (les rhums), enfin les atlas des routes maritimes.

D'abord, quatre chapitres pour replacer la Renaissance dans la continuité et les ruptures par rapport à l'héritage antique de Ptolémée questionné par l'élargissement progressif de l'écoumène. Ce sujet pose la confrontation entre la tradition et la nouvelle réalité actée par les grandes découvertes. L'Imago Mundi traditionnelle est ébranlée. Ptolémée est redécouvert tardivement par sa traduction latine de 1409. L'ouvrage fait sensation, bouleverse les idées géographiques de la fin du Moyen Age (p. 12). Il génère $\mathrm{du}$ bouillonnement intellectuel et invite à énoncer moult questions à propos de la configuration de la terre. Un atlas initial constitué de 27 cartes est rapidement enrichi, complété par de nouvelles cartes. Dès 1500, il a profité de sept éditions successives. Ptolémée est le socle stimulant sur lequel progresse la géographie de la Renaissance, jusqu'à Ortelius (1570) puis Mercator (1578). Entre temps se sont amoncelés itinéraires, journaux de voyages, enquêtes, observations astronomiques et encore quantité de compilations pouvant diffuser des erreurs, voire des images fantastiques et déformées du monde. Les données produites méritent d'être vérifiées, comparées, digérées, triées, classées. Cette tâche prend plus d'un siècle pour atteindre un stade de maturité. D'où le paradoxe suivant: "Les Renaissants ont fait progresser le savoir en regardant en arrière » (p. 28). La diffusion des connaissances est lente, parfois freinée par les rétentions d'informations qui affectent les expéditions. Le progrès nait aussi du travail 
des géographes de cabinet qui ont parfois de géniales intuitions. Dès 1507, et grâce au soutien du duc René II de lorraine, Waldseemüller fait paraître sa Cosmographia Introductio. Audacieusement, il écrit de Saint-Dié : «Une quatrième partie du monde a été découverte par Amerigo Vespucio ». Pour quelques créateurs de génie, déplorons cependant beaucoup d'épigones. Dans la saga des découvreurs se profilent Colomb, Magellan, Pinzón, Cabral et encore Henri le navigateur pour le rôle d'impulsion qu'il sut donner. Ils sont soutenus par les grandes puissances maritimes seules capables de financer des projets aussi risqués. C'est le Portugal réuni à l'Espagne en 1580, l'Espagne, puis Rome, Florence, Venise, etc. S'ensuit un glissement opéré en direction des pays septentrionaux. Ces derniers voient dans les cartes des œuvres utilitaires, des instruments de conquête (outil politique, diplomatique et militaire). La carte offre du pouvoir de persuasion et dessine un nouvel espace mental. La production est réalisée à Anvers, Nuremberg, Bâle, Venise, Amsterdam, Londres, etc. Elle évolue en établissant une grammaire des représentations, des symboles, des figurés. Avec la rose des vents et la direction de l'orientation naissent des conventions. Au XVI ${ }^{\mathrm{e}}$ siècle, sous l'influence de l'usage de la boussole, le Nord s'impose en haut. Un laborieux travail de corrections des tracés des continents s'opère. Des efforts de nomenclature s'affichent, avec en abondance, l'usage à des références religieuses pour donner des noms aux terres vierges, par exemple Santa-Cruz, Conception, etc.

5 En second, les chapitre V à IX dressent un état des lieux à propos du rythme irrégulier imprimé aux progrès de la cartographie. Au cours de la Renaissance, ce renouvèlement est à rapprocher du début de la maitrise de la trigonométrie. En résulte la Cosmographie d'Apian (1524). Ce succès éditorial connut soixante éditions. L'évolution passe par la maitrise de la sphère qui est « univers des choses» (p. 107), sans commencement ni fin, mais avec la présence des antipodes. En publiant à Bâle sa Cosmographie (1544), Sébastien Münster (1489-1552) tente de donner un miroir du monde. Montaigne y vit surtout un beau guide de voyage pour organiser ses étapes de cures. Le travail de Munster est le point de départ d'une floraison de publications géographiques dressées dans différentes échelles complémentaires. Il met à la mode les portraits de ville ou vues en perspectives cavalières. Elu parmi les pères de la géographie, $\mathrm{S}$. Munster en établit ses lois, créé un modèle descriptif. Ses héritiers intègrent l'héliocentrisme suite aux découvertes de Galilée, Copernic, Tycho-Brahé qui inaugurent la révolution mécaniste. Ces acquis sont valorisés, par exemple chez les Français Belleforest et André Thevet. Ces auteurs commencent également à présenter les lois et coutumes des peuples. Ainsi, Thevet fait connaître le tabac popularisé par la suite par Nicot (p. 147). La géographie devient un outil de gouvernement. N. Broc étudie les cartes dans les échelles respectives de la cosmographie, de la géographie, de la chorographie. Son principal terrain d'études est l'Allemagne. Il est soutenu par de prestigieux mécènes : les Fugger, Welser, Peutinger. Débutent également des travaux cartographiques régionaux, par exemple avec la représentation de la Suisse dès 1538, la carte des Flandres de Mercator (1540), le Berry (1567), le Beaujolais (1573), la carte de Bavière d'Apian qui bénéficie d'une véritable triangulation (1554). Ajoutons les enquêtes diligentées par Philippe II d'Espagne pour connaitre le potentiel offert par le royaume de Castille (1575-1580). L'Europe des Humanistes s'arrête à l'Est de la Pologne et au Nord du Danemark. Au-delà, l'espace demeure flou, peu visité, craint.

6 Le $\mathrm{XVI}^{\mathrm{e}}$ siècle fut plein de controverses et contradictions, avec des balbutiements et des allers et retours dans la maitrise des connaissances. Les contemporains sont fréquemment écartelés entre l'héritage antique et l'ouverture au monde. Trois types de 
cartes créent des approches complémentaires pour percevoir le monde : les cartes cadastrales ou plans-terriers, les cartes-itinéraires utilisées par les marchands et pèlerins, les cartes "particulières" qui s'efforcent de remplir les espaces rétrolittoraux. S'ajoutent à ces documents des atlas. Le plus connu est le Theatrum d'Ortelius (1570).

7 Les chapitres IX et X osent la confrontation entre ancien et nouveau monde. Il en ressort que la pensée du géographe de la Renaissance emprunte des chemins capricieux. S'y intriquent le réel et l'imaginaire, le respect des textes anciens et l'audace de s'en servir pour aller plus de l'avant et créer de nouvelles hypothèses. Cette posture amène à constater des écarts de temps entre la conquête intellectuelle de la terre et les voyages réels qui servent in fine à valider des hypothèses. Les découvertes et l'euphorie qu'elles entrainent fonctionnent jusque vers 1540-1550.Les grands voyages modifient les regards sur l'ancien monde et ses marges : le Proche Orient, la Perse, et plus loin vers l'Afrique. A partir des comptoirs égrenés par la thalassocratie portugaise, on évoque l'empire du Prêtre Jean, le mythique Monomotapa, nouveau Pérou où l'on espère trouver l'or, l'ivoire, l'ambre, la gomme. Partiellement, la synthèse sur l'Afrique est établie par Jean-Léon qui fut intermédiaire entre Chrétienté et Islam (p. 238). Plus loin, les continents sont seulement effleurés. Les océans sont mieux connus que les terres, en particulier les marées, les courants, les vents étésiens liés aux alizées, les vents de mousson. Dans l'océan Indien et le Pacifique, les jonques chinoises, les praos malais et les caravelles commencent à se croiser (p. 245). Cette ouverture du monde passionne Rome qui eut un pape géographe (le futur Pie II avait compilé une Cosmographie avant d'accéder au trône de Saint-Pierre en 1458) (p. 26). Dans le cadre de la Contre-Réforme, Rome affiche sa suprématie en étant le centre de documentation géographique le plus actif du monde. Les papes Pie IV et Grégoire XIII font peindre des cartes en fresques sur les murs de leurs palais (p. 322). La ville éternelle dispose d'éminents savants avec les membres de la Compagnie de Jésus. Le concile de Trente amorce leur puissant mouvement missionnaire conservé dans les "Lettres indiennes ", les rapports envoyés à Rome sur l'état des pays visités ou investis. Par exemple le père Ricci apprend le mandarin, débarque à Canton en 1582 puis réside à Pékin en 1600 . L'Amérique reste très inégalement relatée. L'hispanique est bien recensée grâce aux inventaires géographiques, démographiques et économiques dressés par l'administration espagnole. Les terres d'Amérique du Nord sont peu investies si l'on excepte le travail de Samuel Champlain dans l'actuelle Belle Province. Le reste du monde est seulement entre-aperçu et l'imagination va bon train à propos des antipodes qui obéissent à des logiques de symétrie. Ortelius, "le Ptolémée du XVI siècle », a même la prémonition d'une immense Terra Australis, ce qui attisera la curiosité des savants jusqu'aux découvertes des marins du XVIII ${ }^{\mathrm{e}}$ siècle.

8 Les derniers chapitres (XII à XV) évoquent la consolidation de la géographie après avoir assimilé et rapproché l'héritage antique et les grandes découvertes. La fièvre des découvertes a précisé les contours du monde transcrit sur le papier. Cohabitent plusieurs types de projections : cordiformes, carrées, ovales, stéréographiques (p. 278). On aboutit à une représentation de la terre en une seule figure, exempte de discontinuités. La Renaissance correspond au temps des atlas. Celui de Mercator est l'aboutissement de toute sa carrière. Ce projet dure de 1569 à 1590. Des notices historico-géographiques sont placées au recto des cartes. Suivent des atlas nationaux, par exemple le Théâtre François de Bourguereau qui a été étudié par le père de Dainville en 1960. Paraissent également des atlas nautiques, voire des recueils de cartes d'îles. 
Cette abondante production s'effectue principalement à partir d'Anvers où résidait l'imprimeur Plantin, puis à Amsterdam. A compter du début du XVII siècle, les villes de la mer du Nord supplantent Lisbonne, Séville et Venise. Dans le contexte de plus en plus troublé de la fin du siècle, les plans de ville établis en vue oblique ou les plans géométriques remplacent les portraits de ville. Ils montrent le passage des cartes heureuses affichant la prospérité des temps de paix aux cartes utilisées pour attaquer, conquérir. Il s'agit des levées établies par Tassin ou Chastillon pour engager un siège.

La typologie qui vient d'être énoncée souligne la diversité et la richesse de la géographie renaissante. Sur un pas de temps débuté dès le Quattrocento, elle lie son avenir aux mathématiques, à l'optique, l'anatomie, la géométrie (p. 299). Dès 1503, cette rencontre des disciplines fut institutionnalisée à Séville où la Casa de Contractacion croisait les questions économiques, le rassemblement des documents, la fabrique des cartes et encore le fonctionnement d'une école navale (p. 311).

Pour terminer, le livre évoque les empreintes de la Renaissance géographique sur l'art pictural et la littérature. Le mot paysage nait avec la Renaissance, le terme artialisation qui fut initié par Montaigne est également contemporain. La présence de cartes est attestée dans les ateliers des peintres et les boutiques des marchands. Léonard de Vinci associa l'art et la géographie (p. 328). Les grandes découvertes renouvelèrent les thèmes d'inspiration, réveillèrent le sentiment de nature. Elles favorisèrent l'essor des cabinets de curiosités. Les continents furent présentés par des allégories, La perspective et les projections progressèrent. Laïcisation, humanisation et rationalisation gagnèrent les esprits. Enfin, la géographie investit la littérature, souvent via l'utopie (Thomas More) ou les fabuleux voyages (Rabelais dans Pantagruel-1532). Montaigne rédige un chapitre intitulé Des Cannibales.

11 Numa broc a produit une somme scientifique érudite et exhaustive pour traiter son sujet. La Géographie de la Renaissance méritait l'effort de réédition engagé par les éditions du CTHS. Elles se mettent au service d'une redécouverte d'un travail éclairant un temps fort de notre civilisation judéo-chrétienne. Sur un pas de temps de deux siècles s'impose une nouvelle vision de l'espace. Les contemporains sont bénéficiaires d'horizons considérablement agrandis. La planète s'y révèle, parfois par des chemins capricieux. La progression de la géographie s'accommode de rythmes lents ou accélérés. Numa Broc nous fait vivre la conquête intellectuelle et matérielle de la terre. Il a démontré que la Renaissance fut un temps d'éducation à la géographie des élites.

\section{AUTEURS}

\section{JEAN-PIERRE HUSSON}

Université de Lorraine 